\title{
The administration of aerosolised medications in bronchiectasis: the rediscovery of an old method
}

\author{
L. Casali ${ }^{1}$, M.E. Crapa²
}

Monaldi Arch Chest Dis 2011; 75: 3, 155-156.

1 Department of Internal Medicine section of Respiratory Diseases, University of Perugia,

2 Department of Internal Medicine section of Respiratory Diseases, University of Perugia, Italy.

Correspondence: Prof. Lucio Casali, Azienda Ospedaliera S. Maria, Via Tristano di Joannuccio 1, 05100 Terni, Italy; e-mail: lcasali@unipg.it

Bronchiectasis can be defined as a permanent, abnormal enlargement of the bronchi, which occupies a critical site interposed between trachea and pulmonary parenchyma [1].

Bronchiectasis is charaterised by recurrent, chronic and often refractory infections with a load of persistent cough, bronchorroea, episodes of hemoptysis, progressive impairment of pulmonary function and, in the course of time, by a progressive decline of the general conditions. The pathogenesis is complex but substantially is based on frequent episodes of superimposed infections with a consequent development of a widespread inflammation both in the nearest territories and sometimes extended to the whole body [1].

Despite a considerable overlap among the various forms of bronchiectasis, it is possible to distinguish different clinical and radiographic patterns which form the basis for diagnosis and treatment [2].

Given a common inflammatory process several pathways can lead to the development of bronchiectasis and a classification might reflect the following categories [3]: structural lung condition, toxic damage to airways, obstruction of single bronchi, obstructive airways disease, defect of mucociliary clearance, allergic bronchopulmonary aspergillosis, immunodeficiency, infections, bronchiectasis in systemic diseases, idiopathic disease.

Bronchiectasis may be localised or diffuse and these features can reflect a different origin and therefore determine the choice of the treatment. Although proper classifications can elucidate both the aetiology and the pathogenesis, the CT scans can support the hypothetic line of reasoning of the clinician, but the final decision on the treatment may be critical. In fact correct therapeutic strategies lead to important results concerning the health of the patients and the related direct and indirect costs. The economic burden for these disease is heavy because bronchiectasis is chronically marked by a progressive course, requires frequent medical care, prescription of antibiotics and other supportive substances, hospitalisations and chest physiotherapy in order to reduce the number of recurrent infections and slowing the progression of the disease. In a few cases, surgery may be required [4].

Ten years ago it was estimated that annual costs of one case of bronchiectasis in USA was 13.244 USD, greater than that of similar costs for heart diseases and COPD [5]. In that country the global cost has been calculated as greater as 1.4 billion/year [5]. Among all ages it has been estimated that about 25/100.000 people have bronchiectasis, but this number increases to 272/100.000 for those over 74 yrs [6].

The situation can be much more severe when an infection resistant to oral antibiotics is present. In fact the administration of intravenous antibiotics requires either hospitalisation or strict domiciliary monitoring, the placement of a central venous catheter with a pump for a correct release of the drug in accordance with the sensitivity tests and the pharmacological assessment. Moreover blood tests are needed to detect possible side effects and to check a general clinical trend. Otherwise because of the established chronicity of these forms two kinds of treatment should be envisaged: an aetiological treatment (when possible) and another able to prevent exacerbations and complications therefore contributing to slow the progression of disease [7].

If antibiotics remain one of the milestones in the treatment of bronchiectasis, the therapeutic strategies include other categories of drugs which concur in assembling a platform where many integrated roles play interdependently. In this field we quote the roles of muco-active agents [8, 9], steroids for their anti-inflammatory action [10], osmolar agents [11], bronchodynamics, mainly $\beta_{2}$ stimulants and anticholinergics $[12,13]$, antifungal agents [14] and genetic treatments in cases of Cystic Fibrosis (CF) [15]. A part from this last option 
at least a fact has to be mentioned: many substances when orally or intravenously administered can reach the target only in part and therefore their direct effects may be blunt. So the possible side effects may be prevalent in comparison with the clinical success and the risk of eliciting resistances becomes real.

In the current issue of Monaldi Archives for Chest Disease, Dal Negro e Micheletto discuss the role of the "Use of aerosol in bronchiectasis patients" [16] describing in detail the role of the overstated categories of drugs when administered by aerosol. The Authors analyse all the pro and contra according to the literature and to their experience.

Undoubtedly this mean of administration has proven to be very helpful particularly in some circumstances.

Reverting to the topic of antibiotic use, the first line in the fight against the exacerbations the inhalation route allow a right concentration of drug directly in the target and in the closer areas. It seems worthwhile to remember that in non-CF patients Pseudomonas Aeruginosa and Haemophilus Influenzae are mostly present and in the course of exacerbations MDR and hypermutative strains are frequent [17]. Conversely, Streptococcus Pneumoniae, Moraxella Catarrhalis and Burkholderia spp. are less frequently encountered. Otherwise in CF besides Pseudomonas Aeruginosa. and Haemophilus Influenzae, Burkholderia spp can represent one of the major causes of morbidity and mortality but the presences of methicillin resistant Staphilococcus Aureus (MRSA) is rising with a lot of additional problems [18].

It is therefore evident that the concrete possibility of delivering antibiotics by means of inhalation may represent a solid resource in the treatment and management of bronchiectasis of whatever origin.

Of course many technical questions strictly regarding the preparations of drugs, the physical characteristics of the solution to aid the specific performance of the device must first be resolved. The choice of a proper device should be firstly oriented on the patients according not only to their needs but also to their physical, anatomic and psychological peculiarities evaluating in a balanced way all their features [19].

In presence of $\mathrm{CF}$, recent innovations in the field of genetics would open new frontiers in the treatment of these severe condition [20] and hopefully these might support more traditional drugs and devices in the progress of management of this disease.

\section{References}

1. Goddard M. Histopatology of bronchiectasis. European Respiratory Society Monograph 2011; 52: 22-31.

2. Reiff DB,Wells AU, Carr DH, et al. CT findings in Bronchiectasis: limited value in distinguishing between idiopathic and specific types. Am J Roentgenol 1995; $165 ; 261-267$.

3. Bilton D, Jones AL. Bronchiectasis epidemiology and causes. European Respiratory Society Monograph 2011; 52; 1-10.

4. Rosen MJ. Chronic cough due to bronchiectasis. ACCP. Evidence-Based Clinical Practice Guidelines. Chest 2006; 129 (suppl 1): 122-131.

5. Weyckr D, Edesberg J, Oster G, Tino G. Prevalence of economic burden of bronchiectasis. Clin Pulm Med 2005; 12: 205-209.

6. O'Donnell AE. Bronchiectasis. Chest 2008; 134: 815-823.

7. LJ Lobo, MA Zariwala, PG Noone. Ciliary dyskinesias: primary ciliary dyskinesia in adults. Eur Res Monograph 2011; 52: 130-149.

8. Stafanger G, Garne S, Howitz P. The clinical effect on the ciliary motility of oral $\mathrm{N}$-acetylcysteine in patients with cystic fibrosis and primary ciliary dyskinesia. Eur Res J 1988; 1: 161-167.

9. Cole PJ. Inflammation: a two edged sword: the model of bronchiectasis. Eur Res Dis Suppl 1986; 147: 6-15.

10. Wark PA., Gibson PG. Allergic bronchopulmonary aspergillosis: new concepts of pathogenesis and treatment. Respirology 2001; 6: 1-7.

11. Kellett F, Redfern J, Niven RM. Evaluation of nebulized Hypertonic saline (7\%) as an adjunct to physiotherapy in patients with stable bronchiectasis. Resp Med 2005; 99: 27-31.

12. Weintraub SJ, Eschnbacher WL. The inhaled bronchodilators iprotropium bromide and mataproterenol in adults with CF. Chest 1989; 95: 861-864.

13. Sanchez J, Halbrown J, Chernick K. Acute bronchodilator response to a combination of beta-adrenergic and anticholinergic agents in patients with cystic fibrosis. J Pediatr 1992; 120: 486-488.

14. Elphic H, Southern K. Antifungal therapy for allergic bronchopulmonary aspergillosis in people with cystic fibrosis. Coch Dat Sys Rev 2000; 4: CD002204.

15. Laube BL. The expanding role of aerosols in systemic drug delivery, gene therapy and vaccination. Resp Care 2005; 50: 1161-1176.

16. Dal Negro RW, Micheletto C. Use of aerosol in bronchiectasis patients. Monaldi Arch Chest Dis 2011; 72: 185-193.

17. Foweraker E, Wat D. Microbiology of non-CF bronchiectasis. European Respiratory Society Monograph 2011; 52: 68-96.

18. Flaherty K, Herridge MS. Clinical Year In Review II. Lung cancer, sleep apnea, interventional pulmonary disease, cystic fibrosis. Proc Am Soc 2011; 8: 398-403.

19. Laube BL, Jansens HM, de Jongh FHC, et al. What the pulmonary specialist should know about the new inhalation therapy. Eur Resp J 2011; 37: 1308-1331.

20. Ramsey BW, Davies J, Mc Elvaney NG, et al. A FCTR pontientiator in patients with cystic fibrosis and the G551D mutation. NEJM 2011; 365: 1663-1672. 